\title{
ESTUDIOS METODOLÓGICOS DEL EFECTO DEL REGADÍO SOBRE LA CALIDAD DE LAS AGUAS DE LAS MASAS ASOCIADAS A UNA ZONA REGABLE: EL CASO DEL SECTOR B-XII DE LA ZONA REGABLE DEL BAJO GUADALQUIVIR
}

\author{
Martínez Beltrán, J.
}

Centro de Estudios Hidrográficos del CEDEX, Paseo Bajo de la Virgen del Puerto 3, 28005 Madrid, julian.martinezbeltran@cedex.es

\section{Resumen}

El objeto del estudio ha sido determinar los efectos de la agricultura de regadío del Sector B-XII en la detracción de agua del Guadalquivir y en los contenidos de sales y nitratos de sus aguas en el tramo asociado a este sector, así como en el agua subterránea subyacente. La dotación de agua asignada en la campaña 2012-2013 ha sido inferior a la calculada para satisfacer las necesidades de agua de riego de los cultivos y el control de la salinidad de los suelos. La detracción de agua para el riego de este sector está justificada si han de mantenerse sus beneficios socioeconómicos actuales. En el año hidrológico 20122013 el sector exportó más sales que las que entraron con el agua de riego lo que indica que siguen lavándose sales del subsuelo. El impacto sobre la salinidad del agua del río es limitado ya que la mayor masa de sales se evacua en otoño-invierno cuando los caudales del río son mayores y por tanto su capacidad de dilución. La salinidad del agua del río depende más de su caudal y de su efecto sobre la cuña de agua salobre marina que de la evacuación de sales con el agua de drenaje. La masa de nitratos exportada es también mayor que la importada con el agua de riego. Queda por determinar si se debe a nitratos aplicados en la campaña estudiada o de nitratos acumulados en el suelo por exceso de fertilización nitrogenada en campañas anteriores. El riego y el drenaje de las tierras del sector han contribuido a controlar el nivel freático según las necesidades de los cultivos y a disminuir progresivamente la salinidad del agua subterránea.

\section{Introducción}

La agricultura de regadío tiene efectos socio económicos muy relevantes si la zona regable donde se practica ha sido correctamente planificada y diseñada y sus obras bien ejecutadas. Además, los sistemas de riego y drenaje deben ser adecuadamente operados y mantenidos por la comunidad de usuarios y por los propios regantes, que a su vez deben aplicar buenas prácticas agrarias. Sin embargo, un regadío detrae recursos hídricos de una masa de agua y la evacuación del agua de drenaje afecta a la calidad del agua de la masa asociada que lo recibe. Por ello, la Dirección General del Agua (DGA), del Ministerio de Agricultura, Alimentación y Medio Ambiente, encomendó al CEDEX un estudio metodológico de esos efectos en tres zonas regables (con una extensión total de unas 85.000 ha): la zona regable en el delta por el Canal de la Derecha del Ebro, el Sector B-XII de la Zona Regable del Bajo Guadalquivir y la Zona Regable del Canal de Orellana. Este estudio (DGA/CEDEX, 2013) incluye la caracterización de cada zona, los efectos del riego y drenaje sobre las masas de agua asociadas y el uso sostenible del agua.

El objetivo de este artículo es sintetizar los resultados del trabajo realizado en el Sector B-XII, último de la Zona Regable del Bajo Guadalquivir, situado en la provincia de 
Sevilla, siendo Lebrija la sede de su Comunidad de Regantes (CR-BXII). Tiene una superficie de 15.420 ha de las que 14.087 ha son tierras regables.

La transformación de sus tierras para su uso agrícola comenzó en la primera mitad del siglo XX, cuando la Compañía de Marismas del Guadalquivir construyó en la Sección III de Marismas un dique para impedir la entrada de agua exterior, 4 colectores y 2 compuertas de marea para evacuar al río el exceso de agua de lluvia. Tras los estudios edafológicos, hidrológicos y agronómicos de Grande Covián (1956 y 1967), el Instituto Nacional de Colonización (INC) construyó zanjas abiertas de drenaje en el subsector norte y posteriormente sistemas de tubería, una vez comprobado el funcionamiento del drenaje y la desalación de los suelos con agua de lluvia. En 1980, La Confederación Hidrográfica del Guadalquivir (CHG) y el Instituto Nacional de Reforma y Desarrollo Agrario (IRYDA) terminaron la construcción de los sistemas de riego y de drenaje. La Sección III pasó a ser el Sector B-XII de la zona regable, el IRYDA entregó a 1.142 concesionarios parcelas de 12,5 ha y se constituyó la CR-BXII.

Estudios del IRYDA (1984) mostraron que la evacuación del agua por gravedad era insuficiente para controlar el exceso de agua de lluvia en las zonas más bajas del sector. Por ello, construyó 4 estaciones de bombeo y la CHG posteriormente otras dos. Durante las sequías de la década de 1990, el sistema general de regulación de la cuenca no fue capaz de proporcionar las dotaciones de riego asignadas a este sector. Por ello, la CHG terminó en 2002 la construcción del embalse de Melendo con objeto de mejorar la regulación del tramo final del canal del Bajo Guadalquivir.

\section{Características del Sector B-XII}

El clima es mediterráneo con influencia atlántica. La temperatura media anual en Lebrija es de $18,6^{\circ} \mathrm{C}$. Julio tiene las temperaturas medias más elevadas: $35,3^{\circ} \mathrm{C}$ de máxima y $19,4^{\circ} \mathrm{C}$ de mínima. Las más bajas en enero: $15,9^{\circ} \mathrm{C}$ de y $5,2^{\circ} \mathrm{C}$ de mínima. Todos los años se superan los $40^{\circ} \mathrm{C}$ en varias ocasiones. Hay heladas unos 4 días al año de media (CRBXII, 2009).

La precipitación media anual en la estación de La Señuela durante los últimos 36 años fue $494 \mathrm{~mm}$. Las lluvias se concentran de octubre a abril, siendo noviembre y diciembre los meses con mayor precipitación. La variabilidad temporal es muy grande con años con menos de $300 \mathrm{~mm}$ y otros de pluviometría normal e incluso superior a la media (más de $1.000 \mathrm{~mm}$ en el año hidrológico 1995-1996). En invierno puede haber lluvias intensas en períodos cortos de tiempo: $80 \mathrm{~mm}$ en 24 horas y $124 \mathrm{~mm}$ en 3 días consecutivos, para un período de retorno de 10 años.

La evapotranspiración de referencia $\left(E T_{0}\right)$ anual es superior a la precipitación anual. Durante los últimos 13 años en la estación Lebrija I, la $E T_{0}$ fue $1.432,1 \mathrm{~mm}$, con un máximo en julio de $217,5 \mathrm{~mm}(7,0 \mathrm{~mm} / \mathrm{d})$ y un mínimo en diciembre de $39,5 \mathrm{~mm}(1,3 \mathrm{~mm} / \mathrm{d})$. Existe falta de agua de marzo a octubre y exceso de noviembre a febrero.

Las tierras del sector están situadas en la llanura de estuario del río Guadalquivir sobre antiguos bancos (vetas), transiciones, depresiones (lucios) y cauces (caños). Aunque la cota media del terreno es de unos $3 \mathrm{~m}$ sobre el nivel medio del mar, los terrenos de caños y lucios están a 2,5-2,6 m.

Los suelos tienen más del 60 por ciento de arcilla de tipo expansivo, sin arena y en su estado natural eran salinos y sódicos. Las tierras carecían de drenaje natural debido a la baja permeabilidad de los suelos y a una capa impermeable a algo menos de un metro de profundidad. El perfil del suelo muestra una capa laborable de $25-30 \mathrm{~cm}$ con estructura poliédrica, una conductividad hidráulica $(K)$ mayor de $0,6 \mathrm{~m} / \mathrm{d}$ y una porosidad efectiva $(\mu)$ en torno a 0,04 . Por debajo existe un horizonte que llega hasta los $60-70 \mathrm{~cm}$, con estructura prismática, grietas muy desarrolladas en estado seco y valores de $K$ y $\mu$ que decrecen con la profundidad hasta aproximadamente $0,1 \mathrm{~m} / \mathrm{d}$ y 0,01 respectivamente. A partir de $60-70 \mathrm{~cm}$, el subsuelo es compacto con $K$ menor de $0,1 \mathrm{~m} / \mathrm{d}$ (Martínez Beltrán, 1988). 
La profundidad efectiva del suelo (unos $65 \mathrm{~cm}$ ) ahora no es salina pero el subsuelo continúa siéndolo. En dicha profundidad el contenido medio de humedad a capacidad de campo es del 33,5 por ciento y la retención de humedad de unos $220 \mathrm{~mm}$. La retención en el intervalo de agua útil varía entre $50 \mathrm{~mm}$ (IRYDA, 1984) y $60 \mathrm{~mm}$ (Lampe, 1998).

Una masa de agua asociada al sector es el tramo del río Guadalquivir que lo limita por el oeste. Su dique (cota $5 \mathrm{~m}$ ) impide la entrada de agua superficial dándole una configuración de pólder. Las aguas en este tramo son de transición con influencia de mareas. Su caudal, regulado por la presa de Alcalá, Sevilla, varió en el otoño-invierno de $2009-2010$ entre $5,4 \mathrm{~m}^{3} / \mathrm{s}$ y unos $2.900 \mathrm{~m}^{3} / \mathrm{s}$ y en la campaña de riego de 40 a $50 \mathrm{~m}^{3} / \mathrm{s}$ (DGA/CEDEX, 2012).

Durante los períodos de otoño-invierno de los años 2008-2013, el agua de este tramo del río tuvo una conductividad eléctrica $(C E)$ de $1,1 \mathrm{dS} / \mathrm{m}$ con caudales entre 150 y $250 \mathrm{~m}^{3} / \mathrm{s}$. Para caudales de unos $60 \mathrm{~m}^{3} / \mathrm{s}$ varió de 2,6 (aguas arriba) a 3,5-5,2 dS/m (aguas abajo) y para caudales menores de $10 \mathrm{~m}^{3} / \mathrm{s}$ entre 3 y $5,5 \mathrm{dS} / \mathrm{m}$ respectivamente. En primavera para caudales de $10-15 \mathrm{~m}^{3} / \mathrm{s}$ la $C E$ fue respectivamente entre $5-7$ y $10-13 \mathrm{dS} / \mathrm{m}$. Durante las campañas de riego, con caudales superiores a $30 \mathrm{~m} / \mathrm{s}$ la $C E$ varió entre 1,6-3,0 dS/m (aguas arriba) y 2-9 dS/m (aguas abajo) y al final del tramo $9-16 \mathrm{dS} / \mathrm{m}$ para caudales inferiores a $30 \mathrm{~m}^{3} / \mathrm{s}$. Este aumento de la $C E$ es debido a la entrada de agua de mar en el río.

Durante 2008-2013, la concentración de nitratos $\left(\mathrm{NO}_{3}{ }^{-}\right)$del agua varió desde unos 50 $\mathrm{mg} / \mathrm{l}$ al inicio del tramo a $35 \mathrm{mg} / \mathrm{l}$ al final con un caudal de unos $50 \mathrm{~m} / \mathrm{s}$, probablemente debido a que el agua de la cuña salina tiene menor $\mathrm{NO}_{3}{ }^{-}$que la del agua en Alcalá.

Otra masa es de agua subterránea de una capa colgada sobre la capa impermeable. Actualmente, los sistemas de drenaje subterráneo controlan el nivel freático por debajo de la zona radicular de los cultivos. La profundidad de dicho nivel se estimó mediante la ecuación de Boussinesq (1904) para flujo en régimen variable, ya que existe una relación directa entre la carga hidráulica sobre el nivel de los drenes y el caudal del agua de drenaje $\left(q_{d r}\right)$, en función de la $K$ y del espaciamiento entre drenes situados sobre una capa impermeable.

El lavado ha reducido la CE del agua freática desde valores iniciales de 76 a $100 \mathrm{dS} / \mathrm{m}$ (INC, 1970). Si el nivel freático es alto el agua es actualmente (2013) ligeramente salina (CE entre 3 y $4 \mathrm{dS} / \mathrm{m}$ ). Entre 30 y $65 \mathrm{~cm}$ la $C E$ osciló entre 4 y $8 \mathrm{dS} / \mathrm{m}$ y en el subsuelo entre 10 y $15 \mathrm{dS} / \mathrm{m}$. En la capa menos permeable la $C E$ alcanza $28 \mathrm{dS} / \mathrm{m}$. Sin embargo, en esa posición los caudales de drenaje son muy pequeños.

En abril de 2013, la $\mathrm{NO}_{3}{ }^{-}$del agua con nivel freático a unos $70-80 \mathrm{~cm}$ varió entre $70-90$ $\mathrm{mg} / \mathrm{L}$ en una parcela con trigo, $120-260 \mathrm{mg} / \mathrm{L}$ en otra de remolacha y en terrenos preparados para sembrar algodón entre 45 y $50 \mathrm{mg} / \mathrm{L}$. En julio, en parcelas cultivadas de maíz unos 200 $\mathrm{mg} / \mathrm{L}$ en el agua entre $30-45 \mathrm{~cm}$, aumentando a $550-750 \mathrm{mg} / \mathrm{L}$ a $70-80 \mathrm{~cm}$, para ser algo menor (265-425 mg/L) cuando el agua estaba más profunda. A $80-90 \mathrm{~cm}$ en parcelas con tomate entre 265 y $425 \mathrm{mg} / \mathrm{L}$ y en parcelas con algodón entre 180 y $400 \mathrm{mg} / \mathrm{L}$.

El sistema de riego parte del embalse de Melendo $\left(5 \mathrm{hm}^{3}\right.$ de volumen efectivo) donde termina el canal del Bajo Guadalquivir con un caudal máximo de $22 \mathrm{~m}^{3} / \mathrm{s}$. Tres tuberías conducen el agua hasta una cántara y tres canales secundarios ( $A, B$ y $C$ ) la distribuyen a los tres subsectores. Desde estos canales es bombeada mediante 13 estaciones a una red de tuberías enterradas que la conducen a las parcelas (Figura 1).

La CR-BXII opera y mantiene la red de riego desde el embalse hasta las tomas de parcela. La presión que proporciona el bombeo varía entre 30 y $40 \mathrm{~m}$ de columna de agua. Cada tubería secundaria suministra agua a 4 parcelas (caudal nominal de $0,88 \mathrm{Ls}^{-1} \mathrm{ha}^{-1}$ ) y cada dos parcelas disponen de una toma con $22 \mathrm{~L} / \mathrm{s}$. Los agricultores riegan por aspersión con cobertura total, mediante surcos alternos y por goteo. En la campaña de 2013 regaron 6.000-7.000 ha por aspersión, unas 2.000 ha por goteo y el resto por surcos. Aplican dosis de riego de entre 65 y $100 \mathrm{~mm}$ según el método, el cultivo y su fase de desarrollo.

La CE media del agua de riego en marzo es de unos $2 \mathrm{dS} / \mathrm{m}$ y progresivamente disminuye desde $1,7 \mathrm{dS} / \mathrm{m}$ en mayo a $0,9 \mathrm{dS} / \mathrm{m}$ en agosto. La relación determinada entre la CE (dS/m) y los sólidos disueltos totales (SDT en $\mathrm{g} / \mathrm{L}$ ) fue 0,64. En 2009, la $\mathrm{NO}_{3}{ }^{-}$del agua aumentó de marzo a abril-mayo desde 1,65 a 9,4 para disminuir en junio-julio a 7,4 mg/L. 
La red de drenaje consta de 4 colectores que terminan en 4 estaciones de evacuación del agua. Dos discurren paralelos al dique perimetral y sirven de drenes interceptores y de conexión de todas las estaciones. Trazados perpendicularmente a los colectores desaguan primarios a $2.000 \mathrm{~m}$ de distancia entre sí y a estos secundarios distantes entre sí $500 \mathrm{~m}$ que captan el agua de los drenes parcelarios (Figura 1). Los desagües son zanjas abiertas de sección trapecial constante en cada desagüe, su talud está protegido por vegetación natural salvo en algunos secundarios. El mantenimiento de colectores y primarios es responsabilidad de la CR-BXII.

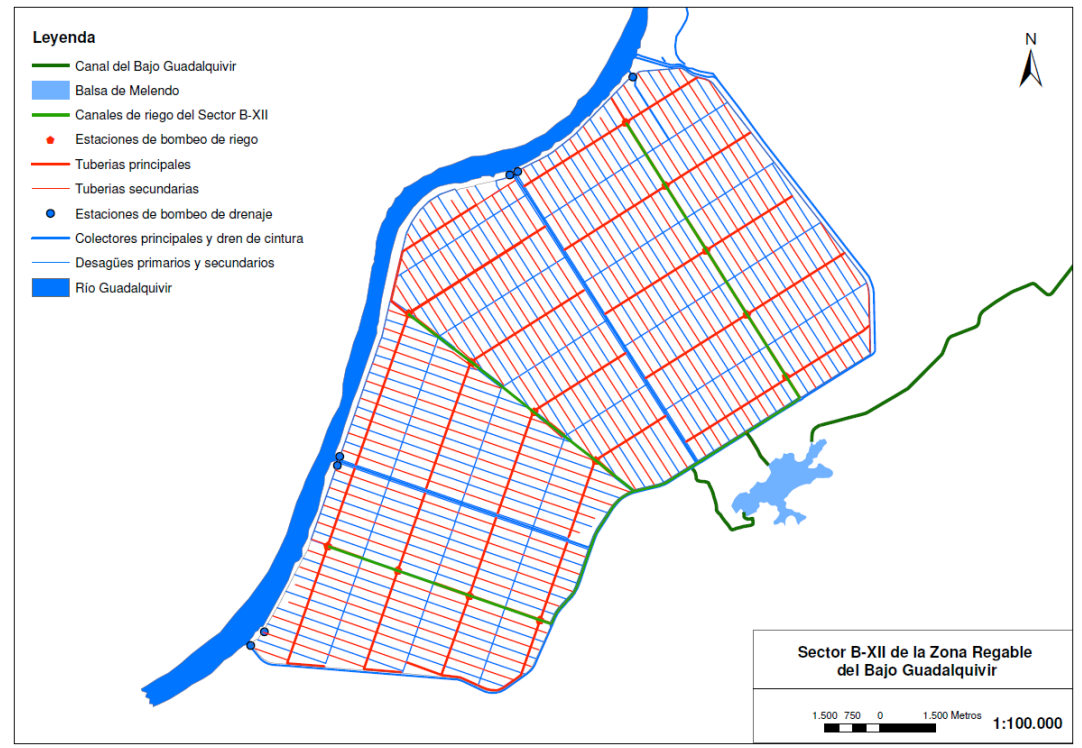

Figura 1. Embalse de Melendo y sistemas de riego y de drenaje del Sector B-XII

Los drenes parcelarios son superficiales, para desaguar la escorrentía superficial del exceso de agua de lluvia, y subterráneos de $250 \mathrm{~m}$ de longitud, de cerámica de $80 \mathrm{~mm}$ de diámetro ( 760 ha con drenes de PVC de $60 \mathrm{~mm}$ ) colocados a una profundidad aproximada de $1 \mathrm{~m}$ y con una pendiente del 1 por mil a espaciamientos de entre 5 y $10 \mathrm{~m}$.

EI IRYDA diseñó sus estaciones para un $q_{d r}$ máximo en la época de lluvias de 12,8 $\mathrm{mm} / \mathrm{d}\left(1,48 \mathrm{Ls}^{-1} \mathrm{ha}^{-1}\right)$ y durante la campaña de riego de $1,2 \mathrm{~mm} / \mathrm{d}\left(0,14 \mathrm{Ls}^{-1} \mathrm{ha}^{-1}\right)$ (Vázquez Guzmán, 1999). La capacidad actual es de $45 \mathrm{~m}^{3} / \mathrm{s}\left(2,92 \mathrm{Ls}^{-1} \mathrm{ha}^{-1}\right.$ y $\left.25,2 \mathrm{~mm} / \mathrm{d}\right)$. Todas las bombas son verticales sumergidas (menos Vetalengua). La altura dinámica máxima de elevación es de $5 \mathrm{~m}$ y la altura geométrica de 3,8 $\mathrm{m}$ (nivel más bajo del agua en el colector en la campaña de riego y nivel del río en pleamar de $4 \mathrm{~m}$ ).

La capacidad y potencia de las bombas varía de $0,75 \mathrm{~m}^{3} / \mathrm{s}$ y $75 \mathrm{CV}$ a $5,0 \mathrm{~m}^{3} / \mathrm{s}$ y 400 CV. La CR-BXII las pone en funcionamiento ante el riesgo de precipitaciones intensas con objeto de bajar el nivel del agua en la red. Durante la campaña de riego opera por la noche una de las bombas de menor capacidad de cada estación. Las bombas más recientes solamente funcionan en momentos de máxima evacuación y las compuertas generalmente permanecen cerradas.

Los cultivos más importantes por su extensión en invierno-primavera son remolacha y trigo. Los agricultores los siembran durante noviembre y cosechan la remolacha en julio con rendimientos medios de 80-90 t/ha. El trigo a final de mayo principios de junio con rendimientos de $6.000-7.000 \mathrm{~kg} / \mathrm{ha}$. En $2012-2013$ cultivaron 1.366 ha de remolacha $(9,8$ por ciento de las 13.916 ha cultivadas esa campaña) y 1.383 ha de trigo (9,9 por ciento). A partir de marzo precisan riego para cubrir su déficit de precipitación.

En primavera-verano: algodón, tomate y maíz. En menor extensión: girasol, alfalfa, zanahoria y cebolla. En 2013 los agricultores cultivaron 6.258 ha (45 por ciento de la superficie cultivada) de algodón. Lo cosechan de octubre a noviembre pero solamente lo riegan por surcos hasta septiembre, pues durante este mes el cultivo absorbe humedad del 
suelo. Sus rendimientos medios varían entre 4.000 y $4.500 \mathrm{~kg} / \mathrm{ha}$. El tomate para uso industrial ocupó 1.645 ha en 2013 (11,8 por ciento). Lo plantan de marzo a abril y lo cosechan durante la segunda quincena de julio y la primera de agosto. Prácticamente en su totalidad lo riegan por goteo. Sus rendimientos pueden alcanzar actualmente $130 \mathrm{t} / \mathrm{ha}$. De maíz cultivaron 1.843 ha (13,2 por ciento). Lo siembran a final de marzo y lo cosechan a final de agosto y principios de septiembre. Lo riegan por surcos y por goteo. Sus rendimientos superan las 12 t/ha de grano. El girasol ocupó 935 ha (el 6,7 por ciento). También lo siembran durante la segunda quincena de marzo y lo cosechan a final de julio.

La rotación habitual es un cultivo de verano (algodón o tomate) seguido al menos cada tres años de uno de invierno-primavera (trigo o remolacha). Así queda garantizado un periodo de tiempo en verano para subsolar en suelo seco.

\section{Balances de agua}

El balance de agua es del año hidrológico 2012-2013 porque el de la campaña anterior es incompleto. Considera el sector en su totalidad, porque todas las estaciones de bombeo están conectadas entre sí por la red de desagües, y las entradas de agua (riego y precipitación) y las salidas (consumo de agua de los cultivos y drenaje del exceso de agua). La diferencia entre entradas y salidas durante el año hidrológico equivale al aumento o disminución de la humedad almacenada en el suelo.

Los resultados de los cálculos para la campaña 2012-2013 muestran los volúmenes mensuales suministrados y evacuados en cada estación y en el conjunto del sector (DGA/CEDEX, 2013) que se presentan convertidos en alturas de lámina de agua (Tabla 1).

Tabla 1. Cantidades de agua de riego suministrada y de agua de drenaje evacuada durante la campaña de riego 2012-2013

\begin{tabular}{|c|c|c|c|c|c|c|c|c|c|c|}
\hline \multirow{2}{*}{ Mes } & \multirow{2}{*}{$\begin{array}{c}\text { Superficie } \\
\text { cultivada } \\
\text { ha }\end{array}$} & \multirow{2}{*}{$\begin{array}{c}\text { Área } \\
\text { B-XII } \\
\text { ha }\end{array}$} & \multirow{2}{*}{$\begin{array}{c}\text { Suministros } \\
\text { de agua } \\
\mathrm{m}^{3}\end{array}$} & \multirow{2}{*}{$\begin{array}{c}\text { Volúmenes } \\
\text { evacuados } \\
\mathrm{m}^{3}\end{array}$} & \multicolumn{2}{|c|}{ Riego } & \multicolumn{3}{|c|}{ Drenaje } & \multirow[b]{2}{*}{$\mathbf{e}_{\mathbf{a}}$} \\
\hline & & & & & $\mathrm{m}^{3} / \mathrm{ha}$ & $\mathrm{mm}$ & $\mathrm{m}^{3} / \mathrm{ha}$ & $\mathrm{mm}$ & $\mathrm{mm} / \mathrm{d}$ & \\
\hline Oct-12 & 587,50 & 15.420 & 0 & 1.014.120 & 0 & 0 & 65,77 & 6,58 & 0,21 & - \\
\hline Nov-12 & $2.360,29$ & 15.420 & 0 & 4.921 .560 & 0 & 0 & 319,17 & 31,92 & 1,06 & - \\
\hline Dic-12 & $2.860,29$ & 15.420 & 0 & 1.289 .520 & 0 & 0 & 83,63 & 8,36 & 0,27 & - \\
\hline Ene-13 & $2.860,29$ & 15.420 & 0 & 1.549 .800 & 0 & 0 & 100,51 & 10,05 & 0,32 & - \\
\hline Feb-13 & $2.860,29$ & 15.420 & 3.167 .118 & 2.801 .880 & $1.107,27$ & 110,78 & 181,70 & 18,17 & 0,65 & 0,84 \\
\hline Mar-13 & $13.585,09$ & 15.420 & 0 & 12.209.760 & 0 & 0 & 791,81 & 79,18 & 2,55 & - \\
\hline Abr-13 & $13.797,79$ & 15.420 & 4.837 .836 & 4.233 .960 & 350,62 & 35,06 & 274,56 & 27,46 & 0,92 & - \\
\hline May-13 & $13.797,79$ & 15.420 & 13.445 .842 & 2.875 .680 & 974,49 & 97,45 & 208,42 & 20,84 & 0,67 & 0,79 \\
\hline Jun-13 & $12.390,79$ & 15.420 & 15.950 .205 & 1.941 .480 & $1.287,26$ & 128,73 & 156,70 & 15,69 & 0,52 & 0,88 \\
\hline Jul-13 & $12.390,79$ & 15.420 & 20.752 .672 & 2.280 .240 & $1.674,85$ & 167,48 & 184,03 & 18,40 & 0,59 & 0,89 \\
\hline Ago-13 & $9.380,12$ & 15.420 & 17.237 .137 & 1.193 .040 & $1.837,62$ & 183,76 & 127,19 & 12,72 & 0,41 & 0,93 \\
\hline Sep-13 & $6.558,20$ & 15.420 & 2.066 .454 & 755.640 & 315,09 & 31,51 & 115,22 & 11,52 & 0,38 & 0,63 \\
\hline Campaña & & & 77.457 .264 & 37.066 .680 & $7.547,22$ & 754,72 & $2.608,70$ & 260,87 & & \\
\hline
\end{tabular}

Los volúmenes de agua aportados se han estimado a partir de los suministros de la CHG a la CR-BXII y a la zona de Toril. Como la superficie del sector supone el 93,4 por ciento, se ha asumido que el sector ha recibido el mismo porcentaje de agua. A este volumen se ha descontado el de sus usos agro-industriales y termo-solares.

La CR-BXII midió mensualmente a partir de mayo de 2012 las horas de funcionamiento de cada uno de los grupos de cada una de las 7 estaciones de bombeo de agua de drenaje. Estimó el caudal que cada grupo podría elevar teniendo en cuenta las 
características de las bombas. El cálculo considera la superficie cultivada durante la campaña de riego, cuando se asume que toda la precipitación es efectiva.

En otoño-invierno con lluvias que producen escorrentía, el cálculo considera toda la superficie intramuros del dique perimetral. Entonces las estaciones bombean un mayor volumen de exceso de lluvia (Figura 2). En verano el volumen de agua de drenaje desciende de mayo a septiembre, por ir decreciendo el área regada y el consumo de los cultivos. Durante los períodos de lluvia el caudal de drenaje $\left(q_{d r}\right)$ es variable y se debe principalmente a drenaje superficial. El $q_{d r}$ medio se mantuvo casi constante de junio a septiembre $(0,53$ $\mathrm{mm} / \mathrm{d}$ en 2012 y $0,51 \mathrm{~mm} / \mathrm{d}$ en 2013), confirmando que la aplicación del agua de riego es muy constante y con alta eficiencia (de junio a agosto $e_{a}$ media de 0,9 ).

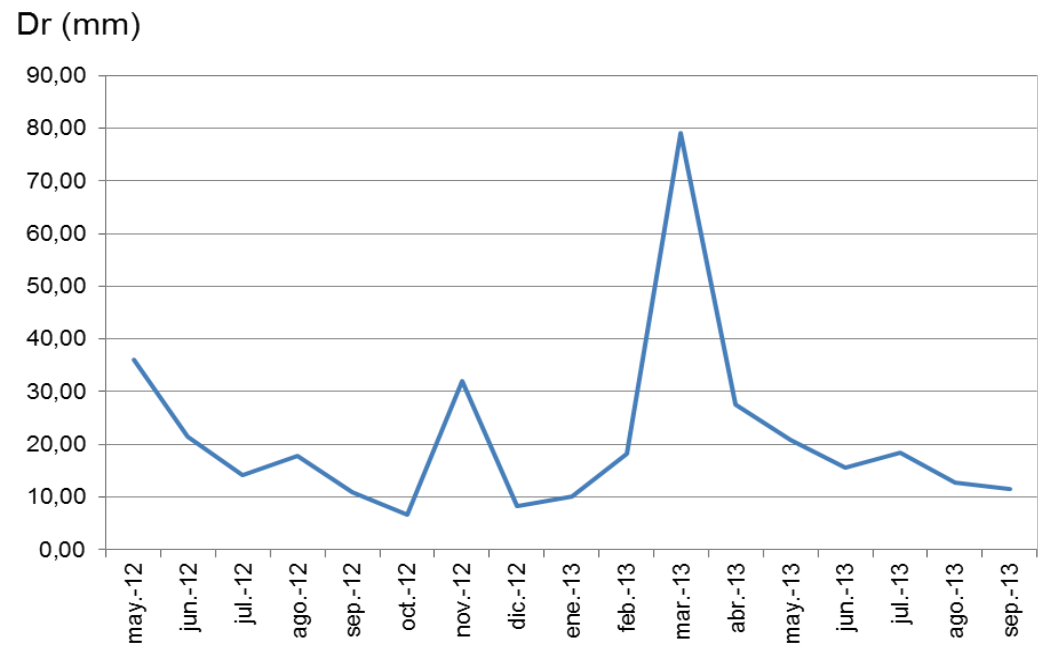

Figura 2. Variación de la lámina de drenaje durante el período estudiado

El cálculo de la precipitación efectiva para los cultivos ha partido de un valor medio de la precipitación media mensual de las estaciones consideradas (DGA/CEDEX, 2013), descontando la escorrentía estimada a partir de los datos de la Tabla 1. En el período considerado sólo se ha producido claramente escorrentía en noviembre de 2012 y de febrero a marzo de 2013 (Tabla 2). Los resultados indican que no hubo escorrentía de agua de lluvia hasta el otoño de 2012 y ésta cesó al comienzo de la primavera de 2013.

Tabla 2. Cálculo de la precipitación efectiva durante el año hidrológico 2012-2013

\begin{tabular}{|c|c|c|c|c|c|c|c|}
\hline \multirow[b]{2}{*}{ Mes } & \multicolumn{3}{|c|}{$P(\mathbf{m m})$} & \multirow{2}{*}{$\begin{array}{c}\mathbf{P} \\
\text { media } \\
\mathbf{m m}\end{array}$} & \multirow{2}{*}{$\begin{array}{l}\text { Drenaje } \\
\text { mm }\end{array}$} & \multirow{2}{*}{$\begin{array}{l}\mathbf{P}_{\text {efectiva }} \\
\mathbf{m m}\end{array}$} & \multirow[b]{2}{*}{$\mathbf{P}_{\mathrm{ef}} / \mathbf{P}$} \\
\hline & Señuela & Lebrija I & Melendo & & & & \\
\hline Octubre 2012 & 111 & 102,6 & 100 & 104,53 & 6,58 & 97,95 & 0,94 \\
\hline Noviembre 2012 & 96,5 & 108,4 & 110 & 104,97 & 31,92 & 73,05 & 0,70 \\
\hline Diciembre 2012 & 27 & 33,8 & 42 & 34,27 & 8,36 & 25,91 & 0,76 \\
\hline Enero 2013 & 52 & 54,4 & 71 & 59,13 & 10,05 & 49,08 & 0,83 \\
\hline Febrero 2013 & 73,5 & 67,8 & 72,5 & 71,27 & 18.17 & 53,10 & 0,75 \\
\hline Marzo 2013 & 132 & 163,3 & 198 & 164,43 & 79,18 & 85,25 & 0,52 \\
\hline Abril 2013 & 75 & 28,2 & 35 & 46,07 & 0 & 46,07 & 1 \\
\hline Mayo 2013 & 2,5 & 7,2 & 3 & 4,23 & 0 & 4,23 & 1 \\
\hline Junio 2013 & 0 & 0 & 0 & 0,00 & 0 & 0,00 & - \\
\hline Julio 2013 & 0 & 0 & 0 & 0,00 & 0 & 0,00 & - \\
\hline Agosto 2013 & 0 & 0 & 0 & 0,00 & 0 & 0,00 & - \\
\hline Septiembre 2013 & 10 & 17,4 & 2 & 9,80 & 0 & 9,80 & 1 \\
\hline $\begin{array}{l}\text { Campaña 2012- } \\
2013\end{array}$ & 579,5 & 583,1 & 633,5 & 598,7 & 154,26 & 444,44 & \\
\hline
\end{tabular}


El cálculo del consumo de agua de los cultivos (Tabla 3) parte de los datos de $E T_{0}$ de la estación Lebrija I y de los coeficientes de cultivo $\left(K_{c}\right)$ de la CR-BXII (2009). Considera la superficie ocupada cada mes por los principales cultivos, que en conjunto representaron en la campaña 2012-2013 el 98,1 por ciento de las tierras agrícolas del sector. El resto incluye el barbecho que prácticamente no consume agua (1,1 por ciento de la superficie).

Tabla 3. Cálculo del consumo de agua durante la campaña 2012-2013

\begin{tabular}{|c|c|c|c|c|c|}
\hline \multirow{2}{*}{ Mes } & \multirow{2}{*}{$\begin{array}{c}\text { Superficie } \\
\text { ha }\end{array}$} & \multicolumn{4}{|c|}{ Consumo } \\
\hline & & $\mathrm{m}^{3}$ & $\mathrm{~m}^{3} / \mathrm{ha}$ & $\mathrm{mm}$ & $\mathrm{mm} / \mathrm{d}$ \\
\hline Octubre 2012 & 587,50 & $248.088,00$ & 422,28 & 42,23 & 1,36 \\
\hline Noviembre 2012 & $2.360,29$ & $424.301,00$ & 179,77 & 17,98 & 0,60 \\
\hline Diciembre 2012 & $2.860,29$ & $323.711,00$ & 113,17 & 11,32 & 0,37 \\
\hline Enero 2013 & $2.860,29$ & $579.263,00$ & 202,52 & 20,25 & 0,65 \\
\hline Febrero 2013 & $2.860,29$ & $1.191 .850,00$ & 416,69 & 41,67 & 1,49 \\
\hline Marzo 2013 & $13.585,09$ & $4.283 .050,00$ & 315,28 & 31,53 & 1,02 \\
\hline Abril 2013 & $13.797,79$ & $11.314 .535,00$ & 820,03 & 82,00 & 2,73 \\
\hline Mayo 2013 & $13.797,79$ & $19.502 .559,00$ & $1.413,46$ & 141,35 & 4,56 \\
\hline Junio 2013 & $12.390,99$ & $26.770 .341,00$ & $2.160,47$ & 216,05 & 7,20 \\
\hline Julio 2013 & $12.390,99$ & $27.178 .388,00$ & $2.193,40$ & 219,34 & 7,08 \\
\hline Agosto 2013 & $9.380,12$ & $15.047 .452,00$ & $1.604,19$ & 160,42 & 5,17 \\
\hline Septiembre 2013 & $6.558,20$ & 4.333.301,00 & 660,75 & 66,07 & 2,20 \\
\hline $\begin{array}{l}\text { Campaña 2012- } \\
2013\end{array}$ & & $111.196 .839,00$ & $10.501,98$ & $1.050,20$ & \\
\hline
\end{tabular}

El balance de agua en el año hidrológico 2012-2013 (Tabla 4) utiliza los valores de las entradas y salidas de agua (Tablas 1-3).

Tabla 4. Balance de agua durante el año hidrológico 2012-2013

\begin{tabular}{|c|c|c|c|c|c|}
\hline \multirow[b]{2}{*}{ Mes } & \multicolumn{2}{|c|}{ Entradas de agua } & \multicolumn{2}{|c|}{ Salidas de agua } & \multirow[b]{2}{*}{$\begin{array}{l}\Delta \mathbf{W} \\
\mathbf{m m}\end{array}$} \\
\hline & $\begin{array}{c}\text { Riego } \\
\text { mm }\end{array}$ & $\begin{array}{l}\text { Precipitación } \\
\text { efectiva } \\
\text { mm }\end{array}$ & $\begin{array}{c}\text { Consumo } \\
\mathrm{mm}\end{array}$ & $\begin{array}{l}\text { Drenaje } \\
\text { mm }\end{array}$ & \\
\hline Octubre 2012 & 0 & 97,95 & 42,23 & 6,58 & 49,14 \\
\hline Noviembre 2012 & 0 & 73,05 & 17,98 & 31,92 & 23,15 \\
\hline Diciembre 2012 & 0 & 25,91 & 11,32 & 8,36 & 6,23 \\
\hline Enero 2013 & 0 & 49,08 & 20,25 & 10,05 & 18,78 \\
\hline Febrero 2013 & 110,78 & 53,10 & 41,67 & 18,17 & 104.04 \\
\hline Marzo 2013 & 0 & 85,25 & 31,53 & 79,18 & $-25,46$ \\
\hline Abril 2013 & 35,06 & 46,07 & 82 & 27,46 & $-28,33$ \\
\hline Mayo 2013 & 97,45 & 4,23 & 141,35 & 20,84 & $-60,51$ \\
\hline Junio 2013 & 128,73 & 0 & 216,05 & 15,69 & $-103,01$ \\
\hline Julio 2013 & 167,48 & 0 & 219,34 & 18,4 & $-70,26$ \\
\hline Agosto 2013 & 183,76 & 0 & 160,42 & 12,72 & 10,62 \\
\hline Septiembre 2013 & 31,51 & 9,8 & 66,07 & 11,52 & $-36,28$ \\
\hline Campaña 2012-2013 & 754,72 & 444,44 & 1050,21 & 260,87 & $-111,92$ \\
\hline
\end{tabular}


Durante la campaña los cultivos utilizaron aproximadamente $112 \mathrm{~mm}$ de la reserva de agua del suelo, casi la mitad de la retención de humedad (unos $220 \mathrm{~mm}$ ). En el otoñoinvierno el suelo aumentó su reserva de agua. A partir de marzo, los cultivos comenzaron a completar sus necesidades de agua con parte de la humedad del suelo. En el otoño no empezó a haber drenaje subterráneo significativo hasta que se repuso cierta cantidad de humedad del suelo: unos $104 \mathrm{~mm}$ de déficit acumulados desde agosto a final de octubre, cifra similar a los 100-120 mm observados en el sector durante los años que lleva cultivándose en regadío. Al nivel de precisión de este estudio, los resultados del balance de agua son lo suficientemente fiables para aceptarlos, así como los volúmenes de agua evacuados estimados por la CR-BXII y utilizados en los siguientes apartados.

\section{Efectos del riego sobre la detracción de agua en el río Guadalquivir}

En la campaña 2012-2013 el consumo de agua de riego de los principales cultivos del sector ascendió a unos $82,8 \mathrm{hm}^{3}$ (Tabla 5). Para una superficie de 13.798 (99,2 por ciento de la cultivada ese año), el consumo medio fue de $6.003,6 \mathrm{~m}^{3} / \mathrm{ha}$. El cálculo de las necesidades de agua de riego en parcela considera una $e_{a}$ de 0,9 (Tabla 1). Podría ser algo mayor en las parcelas regadas por goteo y algo menor en las regadas por surcos y por aspersión. Pérdidas de agua por percolación del 10 por ciento son suficientes para el lavado de las sales aportadas con el agua de riego. Las necesidades medias de agua de riego en parcela serían para la campaña $2012-2013$ de $6.670 \mathrm{~m}^{3} / \mathrm{ha}$.

Tanto para la conducción del agua de riego hasta las estaciones de bombeo como para su distribución se han supuesto eficiencias de 0,95. En el primer caso las pérdidas de agua lo son por evaporación directa en los tres canales del sector pues las de sus aliviaderos son excepcionales. En la distribución posiblemente la eficiencia real sea mayor que la considerada, porque si se producen pérdidas notables de agua la CR-BXII repara las fugas. Con una eficiencia global de conducción-distribución del agua de 0,9 la dotación media de agua de riego para la campaña considerada sería de unos $7.410 \mathrm{~m}^{3} / \mathrm{ha}$. Si la CHG suministró $77,5 \mathrm{hm}^{3}$ a las tierras regables del sector (13.807 ha) en esa campaña de riego, resulta una dotación media disponible de $5.610 \mathrm{~m}^{3} /$ ha claramente inferior a la dotación de la campaña de riego anterior $\left(6.896 \mathrm{~m}^{3} / \mathrm{ha}\right)$ y a la calculada.

Tabla 5. Consumos de agua de los principales cultivos durante la campaña 2012-2013

\begin{tabular}{|c|c|c|c|c|c|c|c|}
\hline \multirow{2}{*}{ Cultivo } & \multirow{2}{*}{$\begin{array}{c}\text { Superficie } \\
\text { ha }\end{array}$} & \multirow{2}{*}{$\begin{array}{l}\mathrm{ET}_{\mathrm{c}} \\
\mathrm{mm} \\
\end{array}$} & \multirow{2}{*}{$\begin{array}{c}\mathbf{P}_{\text {ef }} \\
\text { consumida } \\
\mathrm{mm}\end{array}$} & \multirow{2}{*}{$\begin{array}{c}\mathbf{W} \\
\begin{array}{c}\text { consumida } \\
\text { mm }\end{array} \\
\end{array}$} & \multirow{2}{*}{$\begin{array}{c}\mathrm{I} \\
\mathrm{mm}\end{array}$} & \multicolumn{2}{|c|}{ Consumo parcela } \\
\hline & & & & & & $\mathrm{m}^{3} / \mathrm{ha}$ & $\mathrm{m}^{3}$ \\
\hline Remolacha & $1.365,99$ & 872,14 & 173,79 & 100 & 598,35 & $5.983,5$ & $8.173 .401,17$ \\
\hline Trigo & 1406,8 & 473,88 & 284,89 & 96,5 & 92,49 & 924,9 & $1.301 .149,32$ \\
\hline Algodón & 6258 & 883,20 & 79,74 & 100 & 703,46 & $7.034,6$ & $44.022 .526,80$ \\
\hline Tomate & $1.644,88$ & 754,18 & 84,99 & 100 & 569,19 & $5.691,9$ & $9.362 .492,47$ \\
\hline Maíz & $1.886,80$ & 880,53 & 69,94 & 100 & 710,59 & $7.105,9$ & $13.407 .412,12$ \\
\hline Girasol & 935,12 & 650,12 & 66,66 & 100 & 483,46 & $4.834,6$ & $4.520 .931,15$ \\
\hline Hortalizas & 212,70 & 802,65 & 50,18 & 100 & 652,47 & $6.524,7$ & $1.387 .803,69$ \\
\hline Alfalfa & 87,50 & 1135,52 & 280,76 & 100 & 754,76 & $7.547,6$ & $660.415,00$ \\
\hline Total & $13.797,79$ & & & & & & $82.836 .131,72$ \\
\hline
\end{tabular}

Una primera conclusión es que el riego pudo ser deficitario en la campaña estudiada si los coeficientes $k_{c}$ utilizados fueron correctos y el aporte de humedad del suelo también. 


\section{Efectos del riego y del drenaje sobre la calidad del agua del río Guadalquivir}

El balance de sales durante la campaña 2012-2013 (Tabla 6) parte del balance de agua (Tabla 4). Como aportación de sales considera las aplicadas con el agua de riego y como evacuación las disueltas en el agua de drenaje bombeada al río. Utiliza los datos medios del agua en El Cuenco durante el período 2006-2010 y considera el promedio de los $S D T_{d r}$ en los canales de aproximación a las estaciones de bombeo.

Tabla 6. Balance de sales durante la campaña 2012-2013

\begin{tabular}{|c|c|c|c|c|c|c|c|c|c|}
\hline \multirow{3}{*}{ Mes } & \multicolumn{4}{|c|}{ Sales aportadas } & \multicolumn{4}{|c|}{ Sales evacuadas } & \multirow{3}{*}{$\begin{array}{l}\Delta \boldsymbol{Z} \\
\text { t/ha }\end{array}$} \\
\hline & \multicolumn{2}{|c|}{ Riego } & \multirow{2}{*}{$\begin{array}{c}\mathrm{SDT}_{\mathrm{i}} \\
\mathrm{g} / \mathrm{l}\end{array}$} & \multirow[b]{2}{*}{ t/ha } & \multicolumn{2}{|c|}{ Drenaje } & \multirow{2}{*}{$\begin{array}{c}\mathrm{SDT}_{\mathrm{dr}} \\
\mathrm{g} / \mathrm{l}\end{array}$} & \multirow[b]{2}{*}{ t/ha } & \\
\hline & $\mathrm{mm}$ & $\mathrm{m}^{3} / \mathrm{ha}$ & & & $\mathrm{mm}$ & $\mathrm{m}^{3} / \mathrm{ha}$ & & & \\
\hline Octubre 2012 & 0 & 0 & - & 0 & 6,58 & 65,8 & - & - & - \\
\hline Noviembre 2012 & 0 & 0 & - & 0 & 31,92 & 319,2 & 6,56 & 2,09 & $-2,09$ \\
\hline Diciembre 2012 & 0 & 0 & - & 0 & 8,36 & 83,6 & 8,21 & 0,69 & $-0,69$ \\
\hline Enero 2013 & 0 & 0 & - & 0 & 10,05 & 100,5 & 7,77 & 0,78 & $-0,78$ \\
\hline Febrero 2013 & 110,78 & $1.107,8$ & 1,21 & 1,34 & 18,17 & 181,7 & 8,71 & 1,58 & $-0,24$ \\
\hline Marzo 2013 & 0 & 0 & - & 0 & 79,18 & 791,8 & 5,70 & 4,51 & $-4,51$ \\
\hline Abril 2013 & 5,06 & 350,6 & 1,13 & 0,40 & 27,46 & 274,6 & 6,48 & 1,78 & $-1,38$ \\
\hline Mayo 2013 & 97,45 & 974,5 & 1,06 & 1,03 & 20,84 & 208,4 & 5,54 & 1,15 & $-0,12$ \\
\hline Junio 2013 & 128,73 & $1.287,3$ & 0,81 & 1,04 & 15,69 & 156,9 & 5,04 & 0,79 & 0,25 \\
\hline Julio 2013 & 167,48 & $1.674,8$ & 0,68 & 1,14 & 18,4 & 184 & 3,89 & 0,72 & 0,42 \\
\hline Agosto 2013 & 183,76 & $1.837,6$ & 0,60 & 1,10 & 12,72 & 127,2 & 3,31 & 0,42 & 0,68 \\
\hline Septiembre 2013 & 31,51 & 315,1 & 0,60 & 0,19 & 11,52 & 115,2 & 3,12 & 0,36 & $-0,17$ \\
\hline 2012-2013 & 754,72 & $7.547,2$ & - & 6,24 & 260,87 & $2.608,7$ & - & 14,88 & $-8,64$ \\
\hline
\end{tabular}

El agua de drenaje que llega a las estaciones de bombeo y es evacuada al río Guadalquivir sigue siendo moderadamente salina. En 2013, la CE de esta agua disminuyó desde $13,3 \mathrm{dS} / \mathrm{m}$ en abril a $7,3 \mathrm{dS} / \mathrm{m}$ en julio. En términos de $S D T$ varió durante el año 20122013 de $7,3 \mathrm{~g} / \mathrm{L}$ en otoño-invierno a 5,6 g/L en primavera, descendiendo progresivamente durante la campaña de riego desde $4,7 \mathrm{~g} / \mathrm{L}$ en junio hasta unos $3 \mathrm{~g} / \mathrm{L}$ al final en septiembreoctubre (Figura 4). El gráfico muestra una misma tendencia en las aguas de drenaje que llegan a todas las estaciones de bombeo. Vetalengua en este período no bombeó agua.

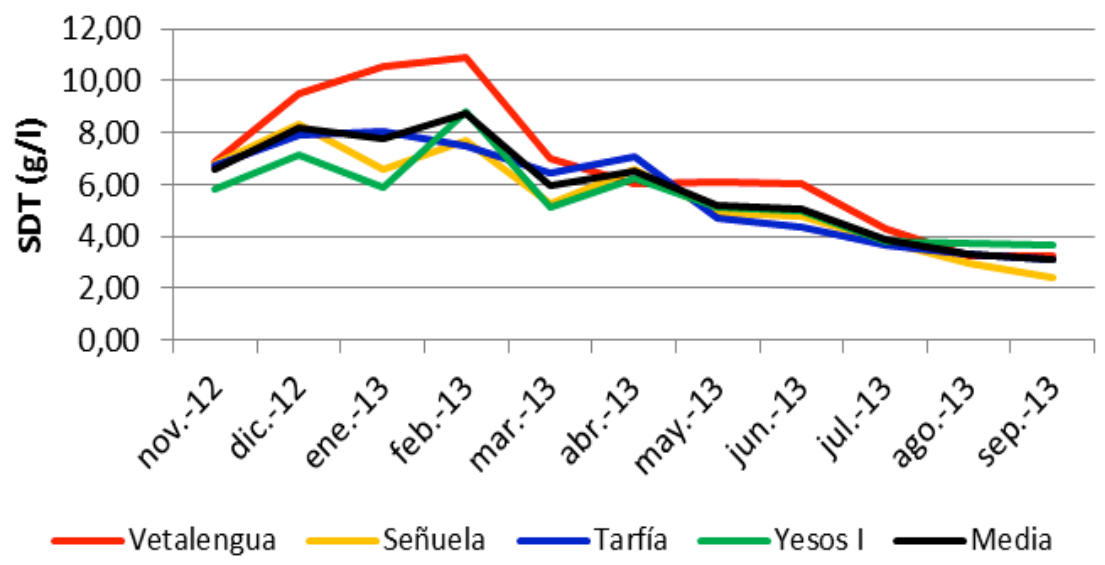

Figura 4. Variación del contenido medio de sales en el agua de drenaje durante la campaña 2012-2013 
El balance indica que las tierras del sector perdieron sales durante dicha campaña $(8,6$ t/ha). Solamente el balance es positivo de junio a agosto, cuando no hay lavado por agua de lluvia y la fracción de lavado es pequeña, por regar los agricultores con una eficiencia muy alta. Las sales acumuladas en esta fase son lavadas posteriormente durante el otoñoinvierno con agua de lluvia y evacuadas por los drenes subterráneos. Esta pérdida de sales se debe a que el subsuelo del sector sigue siendo salino y lo seguirá siendo. La masa de sales evacuadas en cada estación de bombeo y en el conjunto del sector, durante la campaña 2012-2013, se ha calculado multiplicando los volúmenes de agua evacuada cada mes por los SDT en el agua de cada canal de aproximación (Tabla 7 y Figura 5).

Tabla 7. Masa de sales evacuadas en cada estación de bombeo y en el conjunto del sector durante la campaña 2012-2013

\begin{tabular}{|l|r|r|r|r|r|r|}
\hline \multirow{2}{*}{ Mes } & \multicolumn{7}{|c|}{ Masa de sales (t) } \\
\cline { 2 - 7 } & Señuela I & \multicolumn{1}{c|}{ Señuela II } & \multicolumn{1}{c|}{ Tarfía I } & \multicolumn{1}{c|}{ Tarfía II } & \multicolumn{1}{c|}{ Yesos I } & Sector B-XII \\
\hline Octubre 2012 & \multicolumn{1}{c|}{-} & - & - & - & - & \multicolumn{1}{c|}{} \\
\hline Noviembre 2012 & $9.160,32$ & $1.383,93$ & $20.218,57$ & 0 & $2.065,00$ & $\mathbf{3 2 . 8 2 8 , 8 1}$ \\
\hline Diciembre 2012 & $5.157,04$ & 0 & $4.382,46$ & 0 & 806,27 & $\mathbf{1 0 . 3 4 5 , 7 8}$ \\
\hline Enero 2013 & $3.981,66$ & 0 & $5.783,01$ & 968,11 & 554,36 & $\mathbf{1 1 . 2 8 7 , 1 5}$ \\
\hline Febrero 2013 & $8.048,33$ & 58,06 & $9.729,53$ & $1.764,18$ & $1.343,11$ & $\mathbf{2 0 . 9 4 3 , 2 1}$ \\
\hline Marzo 2013 & $21.394,80$ & $4.365,90$ & $32.754,94$ & $8.487,50$ & $3.540,32$ & $\mathbf{7 0 . 5 4 3 , 4 6}$ \\
\hline Abril 2013 & $13.944,50$ & 999,43 & $12.983,59$ & 0 & 867,96 & $\mathbf{2 8 . 7 9 5 , 4 4}$ \\
\hline Mayo 2013 & $7.411,25$ & 0 & $7.236,35$ & 0 & 647,35 & $\mathbf{1 5 . 2 9 4 , 9 5}$ \\
\hline Junio 2013 & $3.153,93$ & 0 & $5.113,77$ & 0 & 551,92 & $\mathbf{8 . 8 1 9 , 5 9}$ \\
\hline Julio 2013 & $3.512,75$ & 26,23 & $3.680,64$ & 0 & 372,34 & $\mathbf{7 . 5 9 1 , 9 6}$ \\
\hline Agosto 2013 & $2.099,91$ & 0 & $1.454,11$ & 0 & 168,29 & $\mathbf{3 . 7 2 2 , 3 1}$ \\
\hline Septiembre 2013 & 872,071 & 0 & $1.129,23$ & 0 & 130,80 & $\mathbf{2 . 1 3 2 , 1 4}$ \\
\hline $\mathbf{2 0 1 2 - 2 0 1 3}$ & $\mathbf{7 8 . 7 3 6 , 5}$ & $\mathbf{6 . 8 3 3 , 5 6}$ & $\mathbf{1 0 4 . 4 6 6 , 2 2}$ & $\mathbf{1 1 . 2 1 9 , 8 0}$ & $\mathbf{1 1 . 0 4 8 , 6 9}$ & $\mathbf{2 1 2 . 3 0 4 , 7 8}$ \\
\hline
\end{tabular}

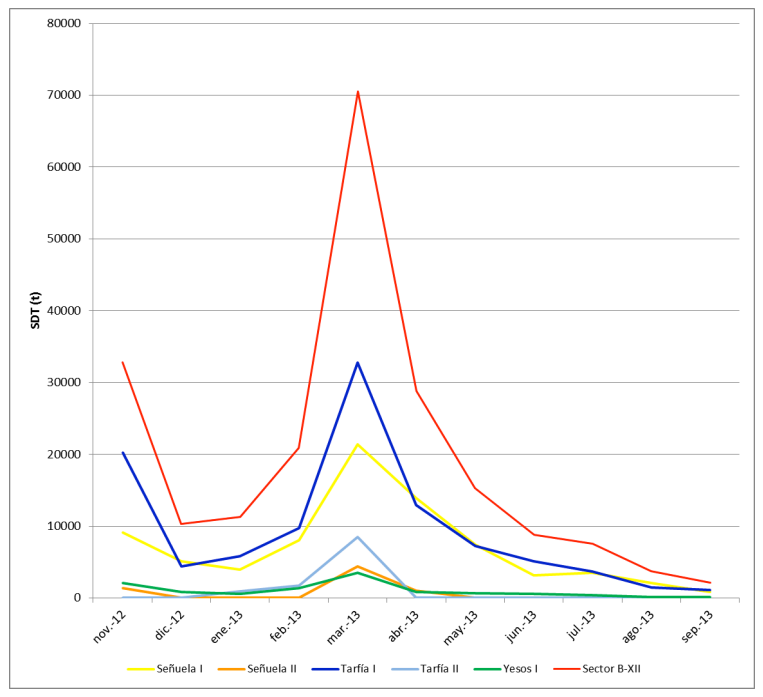

Figura 5. Variación de la masa de sales evacuadas en cada estación de bombeo de agua de drenaje y en el conjunto del Sector B-XII durante la campaña 2012-2013 
Las estaciones Señuela I y Tarfía I evacuaron la mayor cantidad de sales durante la estación de lluvias, coincidiendo con el mayor volumen de agua bombeado, la mayor concentración de sales en el agua de drenaje. Todo ello en la época de mayores caudales del río Guadalquivir y mayor la capacidad de dilución de las sales aportadas. Durante el verano, cuando los caudales del río son menores, tanto el volumen de agua de drenaje como la cantidad de sales bombeadas al río son menores. Sin embargo, la salinidad del agua del río, en las salidas de las estaciones de bombeo, aumenta paulatinamente desde la situada más aguas arriba (Vetalengua) a la que lo está más aguas abajo (Los Yesos). La mayor concentración de sales en el agua se da durante los meses de junio a agosto y la menor de febrero a mayo con un mínimo en abril (Figura 6).

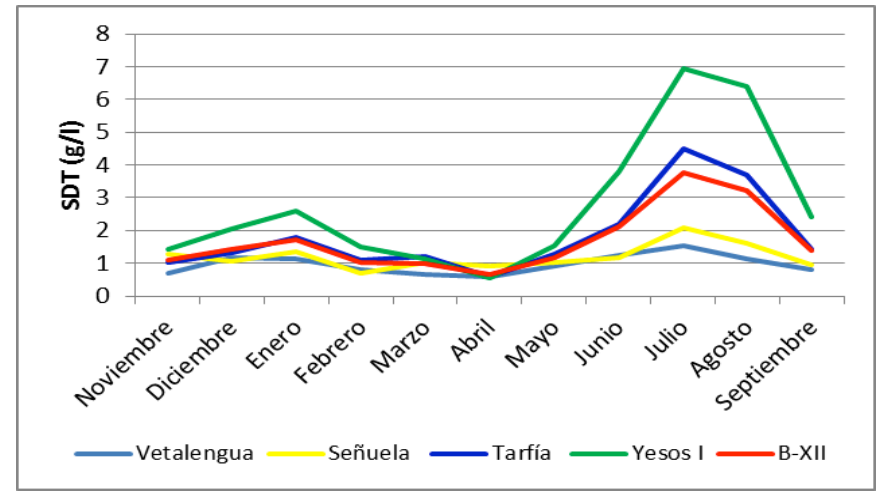

Figura 6. Variación de la salinidad del agua del río Guadalquivir a la salida de cada estación de bombeo de agua de drenaje durante la campaña 2012-2013

Durante las dos campañas estudiadas hay pocos datos de salinidad del agua del río para determinar el efecto de la evacuación de la masa de sales con el agua de drenaje. A mediados de septiembre de 2012, la CE del agua al inicio del tramo del río era $3,5 \mathrm{dS} / \mathrm{m}$ y al final $16,4 \mathrm{dS} / \mathrm{m}$. En septiembre de 2013, los SDT al comienzo del tramo eran de 0,77 g/l y de $5,5 \mathrm{~g} / \mathrm{l}$ al final. La observación de cierta estratificación en la salinidad del agua en este último punto los dos años indica que la influencia de la cuña salina de agua salobre podría tener más influencia en esta variación de la salinidad del agua que la evacuación de sales de este sector, ya que en septiembre la superficie regada es muy pequeña.

Respecto al balance de nitratos los datos disponibles de $\mathrm{NO}_{3}{ }^{-}$del agua de riego son de 2009: en mayo 9,4 mg/L y en junio-julio $7,4 \mathrm{mg} / \mathrm{L}$. Para una dotación media de agua de riego en parcela estimada para la campaña $2012-2013$ en $6.670 \mathrm{~m}^{3} /$ ha y suponiendo una $\mathrm{NO}_{3}{ }^{-}$media de mayo a septiembre de $8 \mathrm{mg} / \mathrm{L}$, la aportación media de nitratos con el agua de riego hubiera sido en dicha campaña de unos $53 \mathrm{~kg} / \mathrm{ha}$. En abril de 2013 la $\mathrm{NO}_{3}{ }^{-}$media de 8 muestras del agua de drenaje que llegaba a las estaciones de bombeo fue $84,9 \mathrm{mg} / \mathrm{L}$ con valores en el intervalo de 50 a $100 \mathrm{mg} / \mathrm{L}$. El valor medio en julio de otras 8 muestras fue 77,9 $\mathrm{mg} / \mathrm{L}$.

Para un volumen medio de agua de drenaje evacuada durante la campaña 2012-2013 de $2.608 \mathrm{~m}^{3} / \mathrm{ha}$ y tomando una $\mathrm{NO}_{3}^{-}$media de $80 \mathrm{mg} / \mathrm{l}$, la cantidad media de nitratos evacuados con el agua de drenaje hubiera sido de unos $208 \mathrm{~kg} / \mathrm{ha}$. Estos resultados, aunque limitados, sí sirven para indicar que este sector exporta más nitratos con el agua de drenaje que los importados con el agua de riego. Es decir, hay un lavado de nitratos de los suelos, bien de los aportados en la fertilización nitrogenada de la campaña estudiada o de los acumulados en el suelo y en el subsuelo, procedentes de campañas anteriores.

Hay pocos datos de $\mathrm{NO}_{3}{ }^{-}$del agua del río Guadalquivir en la campaña 2012-2013: a mediados de septiembre de 2012, la $\mathrm{NO}_{3}{ }^{-}$era al inicio del tramo del río $(6,9 \mathrm{mg} / \mathrm{L})$ y al final $(7,2 \mathrm{mg} / \mathrm{L})$, cifras inferiores a la del agua en la presa de Alcalá $(17,5 \mathrm{mg} / \mathrm{L})$. Valores similares fueron determinados al comienzo de la campaña de 2013-2014 en octubre: respectivamente en los dos puntos mencionados anteriormente 6,9 y $7,7 \mathrm{mg} / \mathrm{L}$. 


\section{Efectos del riego y drenaje de las tierras sobre la capa freática}

El riego y el drenaje de las tierras del sector han afectado al régimen de fluctuación de la capa freática. Los sistemas de drenaje subterráneo controlan ahora la posición del nivel freático. Solamente asciende este nivel a la superficie del terreno durante episodios de lluvias intensas, para descender por efecto del drenaje cuando la intensidad de éste supera a la percolación de agua. Una vez aplicado el riego por surcos o aspersión el nivel freático raramente alcanza la capa laborable. Tras el riego, parte del agua aplicada fluye directamente a la tubería a través de la zanja del dren. Lo más frecuente es que el nivel de agua se sitúe entre 30 y $65 \mathrm{~cm}$ de profundidad. El sistema de drenaje hace descender el nivel freático hasta una profundidad de unos $75-80 \mathrm{~cm}$, justamente por encima del nivel de los drenes. En dos parcelas regadas por goteo, una con maíz y otra con tomate, el nivel freático permaneció a unos $60 \mathrm{~cm}$ de profundidad en julio de 2013.

Con el lavado de los suelos el agua freática también ha ido perdiendo sales, pero sigue siendo salina en contacto con el subsuelo todavía salino. Por ello, los sistemas de drenaje subterráneo seguirán evacuando sales, aunque las $C E$ más altas del agua coinciden con caudales de drenaje inferiores a $0,1 \mathrm{~mm} / \mathrm{d}$.

No hay datos de $\mathrm{NO}_{3}{ }^{-}$del agua freática antes de la transformación en regadío, pero no tendrían que ser altos dado el entonces uso poco intensivo de la tierra. La menor $\mathrm{NO}_{3}{ }^{-}$ determinada en las muestras de agua de 2013 fue $22 \mathrm{mg} / \mathrm{l}$ con nivel freático en la capa laborable y la mayor de $541 \mathrm{mg} / \mathrm{l}$ con el nivel situado a más de $65 \mathrm{~cm}$ de profundidad. Entre estos dos valores extremos la $\mathrm{NO}_{3}^{-}$no solamente depende de la profundidad sino también del cultivo y de la fase de su ciclo relacionada con la práctica del abonado nitrogenado. Estas cifras indican un aumento de la $\mathrm{NO}_{3}{ }^{-}$en el agua subterránea de la capa de agua colgada por efecto de la agricultura de regadío.

\section{Conclusiones y recomendaciones sobre el uso del agua en el sector}

El riego de los cultivos del sector y el drenaje del exceso de agua han sido imprescindibles para la transformación de las tierras de marisma en tierras de uso agrícola. Siguen siendo esenciales para la mejora progresiva de la calidad de los suelos regables pues eliminan progresivamente sales de la capa colgada de agua. La agricultura de regadío del sector es rentable económicamente y se mantiene permanentemente sin deterioro de sus suelos, que progresivamente van perdiendo sales.

La CR-BXII mantiene operativas eficazmente las redes principales de riego y drenaje así como las estaciones adaptando las necesidades de bombeo al consumo mínimo de electricidad. Los regantes aplican el agua con alta eficiencia. Es necesario que mantengan los sistemas de drenaje parcelarios en funcionamiento, con refinos periódicos de los bancales, conservando los tubos de salida de la escorrentía superficial de agua de lluvia a los desagües secundarios y evitando la entrada de raíces en el tramo final de los drenes subterráneos. También conservando la infiltración y permeabilidad del suelo introduciendo al menos cada tres años un cultivo de invierno-primavera con objeto de mantener seco el suelo en verano y practicar un subsolado a $50 \mathrm{~cm}$ cada aproximadamente 3 años.

Las dotaciones de agua suministradas a la CR-BXII por la CHG en las campañas de riego de 2011-2102 y 2012-2013 han sido inferiores a la dotación calculada para satisfacer las necesidades potenciales de agua de los cultivos y el control de la salinidad de los suelos. La detracción de agua del Guadalquivir está plenamente justificada para garantizar los beneficios socioeconómicos que resultan de la agricultura de regadío del sector. Todo ello, para un riego que actualmente es altamente eficiente e incluso posiblemente deficitario.

El manejo actual del agua evita que no se acumulen en las tierras las sales aportadas por el agua de riego. El lavado de sales del subsuelo es progresivo aunque lento y lo seguirá siendo por la reserva de sales que tiene el subsuelo, especialmente al nivel de los drenes y por debajo del mismo. La percolación actual de agua de riego es la mínima para el control 
de la salinidad en la profundidad efectiva del suelo. Por ello, es recomendable mantener la actual eficiencia de riego. El sector evacua la mayor parte de las sales lavadas de sus suelos en la época de mayores caudales del río Guadalquivir, cuando la capacidad de dilución de las sales aportadas es mayor. La salinidad del agua del río Guadalquivir en el tramo asociado al sector B-XII depende más del caudal del río y su efecto en la cuña salina que de la evacuación de sales con el agua de drenaje de este sector.

La masa de nitratos evacuada con el agua de drenaje en la campaña estudiada supera a la masa importada con el agua de riego. El agua de la capa colgada es más rica en nitratos que el agua de riego. Es posible que el abonado actual se adapte a las necesidades de fertilización de los cultivos y que se estén eliminando nitratos acumulados en campañas anteriores, pero esta suposición necesita ser confirmada con un estudio detallado del balance de nitratos en este sector.

Es recomendable determinar las necesidades de agua de los cultivos más relevantes del sector con los datos de la $E T_{0}$ de la estación Lebrija I y revisando los coeficientes $k_{c}$. Asimismo, estudiar la productividad del agua para determinar la dotación de agua más adecuada, con datos de las campañas de riego posteriores a la considerada en este estudio. Tanto para campañas con suministro normal de agua de riego como para riego deficitario en caso de sequías. También estudiar la fertilización nitrogenada de los cultivos determinando las necesidades de abonado y considerando el aporte de nitratos con el agua de riego, de forma que el balance de nitratos en este sector sea equilibrado, con objeto de reducir paulatinamente la masa de nitratos a evacuar con el agua de drenaje bombeada al río Guadalquivir. Además incluir los nitratos en el programa actual de la CR-BXII que determina la salinidad del agua de drenaje que llega a las estaciones de bombeo. Si los agricultores logran equilibrar el balance de nitratos, el exceso almacenado en el subsuelo se irá eliminando gradualmente como ocurre con la salinidad.

Para confirmar las conclusiones de este estudio es también recomendable elaborar balances de agua, sales y nitratos en más campañas de riego, con objeto de obtener valores medios más representativos que los de una única campaña analizada en este estudio.

\section{Agradecimientos}

A la DGA y al CEDEX por la oportunidad de llevar a cabo el estudio de referencia (2013). A Benito Bellido, ingeniero responsable de los servicios técnicos de la CR-BXII, por su información permanente sin la que hubiera sido imposible llevar a cabo este trabajo. A Julia Espina Argüello, de la Consejería de Medio Ambiente de la Junta de Andalucía, por los datos de calidad del agua del Bajo Guadalquivir proporcionados.

\section{Referencias bibliográficas}

Boussinesq (1904). Recherches théoriques sur l'écoulement des nappes d'eau infiltrées dans le sol et sur le dé des sources. Journal des Mathématiques Pures et Appliqués 10-1-78. CR-BXII (2009). Documentación técnica para la inscripción en el registro de aguas del aprovechamiento de la Comunidad de Regantes del Sector B-XII del Bajo Guadalquivir. DGA/CEDEX (2012). Anuario de aforos 2009-2010. Confederaciones Hidrográficas. Dirección General del Agua y CEDEX, Madrid.

DGA/CEDEX (2013). Gestión sostenible del agua de riego en zonas de presiones significativas (detracción/contaminación) sobre masas de agua; estudio de 3 zonas piloto (85.000 ha). Actuación $\mathrm{n}^{\circ} 22$ de la Asistencia Técnica, Investigación y Desarrollo Tecnológico en Materias de Competencia de la Dirección General del Agua (2007-2011).

Grande Covián, R. (1956). Los suelos salinos; su rescate y aplicación a las Marismas del Guadalquivir. Publicaciones del Ministerio de Agricultura, Madrid.

Grande Covián, R. (1967). Las Marismas del Guadalquivir y su rescate. Serie de Estudios del Instituto Nacional de Colonización, volumen V 29, Madrid. 
INC (1970). Estudio de Reconocimiento de Suelos de la Sección III de Marismas del Guadalquivir, Sevilla. Servicio de Suelos del Instituto Nacional de Colonización, Madrid. IRYDA (1984). Evaluación de los sistemas de drenaje de las Marismas del Guadalquivir. Instituto Nacional de Reforma y Desarrollo Agrario, Madrid.

Lampe, U. (1998). Influence of Irrigation and Drainage on Water and Salt Dynamics in Reclaimed Heavy Clay Soils of the Guadalquivir Marshlands, South Spain. Diplomarbeit and der Technischen Universität Carolo Wilhelmina zu Braunschweig. Alemania.

Martínez Beltrán, J. (1988). Drainage criteria for heavy soils with a shallow impervious layer. Agricultural Water Management, 14, 91-96.

Vázquez Guzmán, A. (1999). Saneamiento y drenaje: construcción y mecanización. Editorial Agrícola Española. Madrid. 